\title{
Lectin microarray technology identifies specific lectins related to lymph node metastasis of advanced gastric cancer
}

\author{
Keishi Yamashita ${ }^{1}$ - Atsushi Kuno ${ }^{2}$ Atsushi Matsuda ${ }^{2} \cdot$ Yuzuru Ikehata $^{2}$ • \\ Natsuya Katada ${ }^{1}$ Jun Hirabayashi ${ }^{2} \cdot$ Hisashi Narimatsu ${ }^{2} \cdot$ Masahiko Watanabe $^{1}$
}

Received: 14 December 2014/Accepted: 13 March 2015/Published online: 4 April 2015

(C) The International Gastric Cancer Association and The Japanese Gastric Cancer Association 2015

\begin{abstract}
Background Although various molecular profiling technologies have the potential to predict specific tumor phenotypes, the comprehensive profiling of lectin-bound glycans in human cancer tissues has not yet been achieved. Methods We examined 242 advanced gastric cancer (AGC) patients without or with lymph node metastasisN0 $(n=62)$ or N+ $(n=180)$-by lectin microarray, and identified the specific lectins highly associated with AGC phenotypes.

Results In seven gastric cancer cell lines, in contrast to expressed-in-cancer lectins, not-expressed-in-cancer (NEC) lectins were tentatively designated by lectin microarray. Binding signals of the specific lectins were robustly reduced in AGC patients with $\mathrm{N}+$ status as compared with those with N0 status. The receiver operating characteristic curve determined the optimal cutoff value to differentiate $\mathrm{N} 0$ status from $\mathrm{N}+$ status, and subsequent profiling of NEC lectins identified Vicia villosa agglutinin (VVA) association with the significant other lectins involved in lymph node metastasis. VVA reaction was clearly found on cancer cells, suggesting that it may result
\end{abstract}

Electronic supplementary material The online version of this article (doi:10.1007/s10120-015-0491-2) contains supplementary material, which is available to authorized users.

Keishi Yamashita

keishi23@med.kitasato-u.ac.jp

1 Department of Surgery, Kitasato University School of Medicine, Asamizodai 2-1-1, Minami-ku, Sagamihara, Kanagawa 252-0380, Japan

2 Research Center for Medical Glycoscience, National Institute of Advanced Industrial Science and Technology, AIST Tsukuba Central 2, Tsukuba, Ibaraki 305-8568, Japan from carcinoma-stroma interaction in primary AGC, because VVA is an NEC lectin. Most intriguingly, VVA reaction was remarkably attenuated in the tumor cells of the metastatic lymph nodes, even if it was recognized in primary AGC. In AGC, histological type was strongly associated with soybean agglutinin and Bauhinia purpurea lectin, whereas p53 mutation was the best correlated with Griffonia simplicifolia lectin II.

Conclusions Lectin microarrays can be used to very accurately quantify the reaction of glycans with tumor tissues, and such profiles may represent the specific phenotypes, including $\mathrm{N}+$ status, histological type, or p53 mutation of AGC.

Keywords Lectin array - Gastric cancer - Lymph node metastasis - Vicia villosa agglutinin - Prognosis

\section{Introduction}

Mammalian cells are covered with a dense and complex array of glycans. Different cell types express distinct sets of glycans, and glycosylation of a single cell type significantly changes during malignant transformation $[1,2]$. Certain types of glycan structures are epitope markers for cancer progression, such as sialyl Lewis ${ }^{\mathrm{X}}$ and sialyl Lewis ${ }^{\mathrm{A}}$ (carbohydrate antigen 19-9, CA19-9). Lectins have long been used to characterize cell-surface glycans because of their substantial selectivity [3]. They are also effective in a variety of biochemical and cell biological assays [4, 5]. Although carbohydrate arrays yield valuable data on carbohydrate-interacting proteins, they cannot directly assess changes in glycosylation. Other technologies available for glycan analysis, such as chromatography and mass 
spectrometry, tend to be time-consuming and less suitable for high-throughput evaluation of protein glycosylation. For these reasons, the use of lectins in an array format has gained increased interest [4, 6, 7].

Gastric cancer is one of the leading causes of cancer deaths worldwide. Lymph node metastasis (LNM) is a critical factor affecting the prognosis of gastric cancer [812]. The prediction of LNM would therefore lead to clinical applications. Gene expression profiles determined by DNA microarray analysis predict LNM with about $80 \%$ sensitivity and specificity [13, 14]; however, RNA analysis is not an optimal assay for clinical samples owing to the instability of RNA.

In the present study, we comprehensively analyzed the glycan profiles of cancer tissues to predict specific phenotypes in advanced gastric cancer (AGC). This is the first report to identify critical sets of glycans with altered expression in AGC and to associate the glycan profiles with specific phenotypes.

\section{Materials and methods}

\section{Patients and primary gastric cancer tissues}

The patient characteristics are shown in Table 1. The study subjects consisted of 242 AGC patients who underwent gastrectomy at the Kitasato University Hospital between 1991 and 2000. AGC is defined as gastric cancer in which the depth of invasion reaches the muscularis propria or beyond [15].

Sample sections with a thickness of $5 \mu \mathrm{m}$ were prepared from archival formalin-fixed, paraffin-embedded tissue blocks. Hand-assisted macrodissection of the carcinoma tissues was performed, where the tissue surface was used (Fig. 1a), because we expected to apply this technique for biopsy specimens in the future. Clinical sample collection was approved by the Ethical Committee of Kitasato University School of Medicine.

\section{Cell lines}

The gastric cancer cell line MKN7 was kindly provided by the Cell Resource Center for Biomedical Research Institute of Development, Aging and Cancer, Tohoku University. Six other gastric cancer cell lines (GCIY, AZ521, KE97, SH10, MKN74, and KATOIII) were purchased from RIKEN BioResource Center (Ibaraki, Japan). All cell lines were grown in RPMI 1640 medium (GIBCO, Carlsbad, CA, USA) supplemented with $10 \%$ fetal bovine serum.
Table 1 Characteristics of 242 advanced gastric cancer patients

\begin{tabular}{|c|c|}
\hline Characteristic & Value \\
\hline \multicolumn{2}{|l|}{ Sex } \\
\hline Male & 165 \\
\hline Female & 77 \\
\hline Average age \pm SD (years) & $60.9 \pm 11.8$ \\
\hline \multicolumn{2}{|l|}{ Lauren classification } \\
\hline Diffuse type & 168 \\
\hline Intestinal type & 74 \\
\hline \multicolumn{2}{|l|}{ Portion } \\
\hline Upper & 124 \\
\hline Middle & 54 \\
\hline Lower & 64 \\
\hline \multicolumn{2}{|l|}{ pT status } \\
\hline pT2 & 12 \\
\hline pT3 & 106 \\
\hline pT4a & 103 \\
\hline pT4b & 21 \\
\hline \multicolumn{2}{|l|}{ pN status } \\
\hline pNo & 63 \\
\hline $\mathrm{pN} 1$ & 34 \\
\hline $\mathrm{pN} 2$ & 44 \\
\hline $\mathrm{pN} 3 \mathrm{a}$ & 50 \\
\hline $\mathrm{pN} 3 \mathrm{~b}$ & 51 \\
\hline \multicolumn{2}{|l|}{ fM status } \\
\hline fM0 & 205 \\
\hline fM1 & 37 \\
\hline \multicolumn{2}{|l|}{ UICC stage (7th edition) } \\
\hline IB & 7 \\
\hline IIA & 37 \\
\hline IIB & 32 \\
\hline IIIA & 30 \\
\hline IIIB & 40 \\
\hline IIIC & 59 \\
\hline IV & 37 \\
\hline \multicolumn{2}{|l|}{ JCGC stage (14th edition) } \\
\hline IB & 6 \\
\hline IIA & 34 \\
\hline IIB & 26 \\
\hline IIIA & 24 \\
\hline IIIB & 22 \\
\hline IIIC & 17 \\
\hline IV & 113 \\
\hline \multicolumn{2}{|l|}{ Cytology findings } \\
\hline Negative & 162 \\
\hline Positive & 80 \\
\hline
\end{tabular}

fM status is composed of distant metastasis, peritoneal dissemination, and extraregional lymph node metastasis.

JCGC Japanese Classification of Gastric Cancer, $S D$ standard deviation, UICC Union for International Cancer Control 


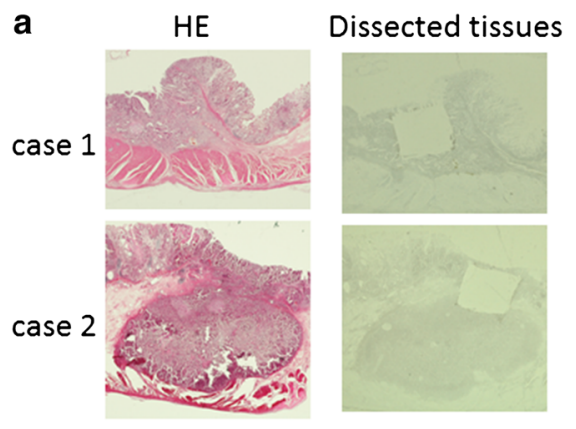

C

\begin{tabular}{|c|c|c|c|}
\hline Marker & 1. LTL & 16. GSL-II & 31. Jacain \\
\hline & 2. PSA & 17. NPA & 32. PNA \\
\hline & 3. LCA & 18. $\operatorname{con} A$ & 33. WFA \\
\hline & 4. UEA-I & 19. GNA & 34. $A C A$ \\
\hline & 5. AOL & 20. HHL & 35. MPA \\
\hline & 6. AAL & BSA & 36. HPA \\
\hline & 7.MAL & BSA & 37. WA \\
\hline & 8. SNA & 23. $\mathrm{BPL}$ & 38. DBA \\
\hline & 9. SSA & 24. TSAII & 39. SBA \\
\hline & 10. TSAI & 25. EEL & 40. GSL-I \\
\hline & 11. PHAL & 26. ABA & 41. PTL-I \\
\hline & 12. ECA & 27. LEL & 42. MAH \\
\hline \multicolumn{3}{|c|}{ 13. RCA120 } & 43. WGA \\
\hline \multicolumn{3}{|c|}{ 14. PHA.E } & 44. GSL-1 A \\
\hline & 15. DSA & 30. PWM & 45. GSL-1B, \\
\hline
\end{tabular}

Fig. 1 Hand-assisted macrodissection and lectin microarray. a Tissue samples (case 1 and case 2) 4-5 mm square were retrieved as in the dissected tissues (right images) with a white background. b Lectin array flow. c The lectin arrangement is shown in the array spots. d Representative lectin microarray spots for gastric cancer tissues. AAL Aleuria aurantia lectin, ABA Agaricus bisporus agglutinin, ACA Amaranthus caudatus agglutinin, AOL Aspergillus oryzae L-fucosespecific lectin, BPL Bauhinia purpurea lectin, BSA Bandeiraea simplicifolia agglutinin, ConA concanavalin A, DBA Dolichos biflorus agglutinin, DSA Datura stramonium agglutinin, ECA Erythrina crista-galli agglutinin, EEL Euonymus europaeus lectin, GNA Galanthus nivalis agglutinin, GSL-I Griffonia simplicifolia lectin I, GSL-I $A_{4}$ Griffonia simplicifolia lectin I isoform A4, GSL-I $B_{4}$ Griffonia simplicifolia lectin I isoform B4, GSL-II Griffonia

\section{Staging system and cytology test}

Staging was performed according to the latest edition, the 14th edition, of the Japanese Classification of Gastric Cancer. The cytology test is a staging test that is unique to the Japanese Classification of Gastric Cancer, and patients are designated as having stage IV gastric cancer if the cytology test result is positive [15].

\section{Fluorescent staining}

Fluorescently labeled proteins were prepared as previously described [16, 17]. Briefly, macrodissected tissue samples (about $5 \mathrm{~mm}$ square) were collected in $10 \mathrm{mM}$ citrate buffer ( $\mathrm{pH}$ 6.0) and incubated for $1 \mathrm{~h}$ at $95{ }^{\circ} \mathrm{C}$ (antigen retrieval). The solution was then centrifuged at $20,000 \mathrm{~g}$ for b

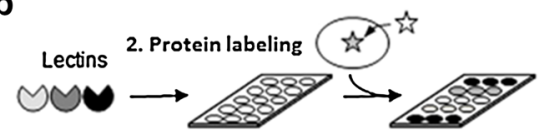

1.Immobilization 3. Protein binding

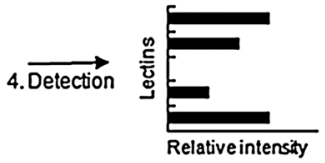

d

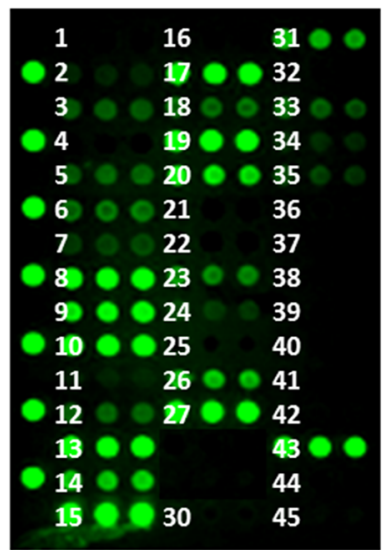

simplicifolia lectin II, $H E$ hematoxylin and eosin, HHL Hippeastrum hybrid lectin, HPA Helix pomatia agglutinin, LCA Lens culinaris agglutinin, LEL Lycopersicon esculentum lectin, LTL Lycopersicon esculentum lectin, MAH Maackia amurensis hemagglutinin, MAL Maackia amurensis lectin, MPA Maclura pomifera agglutinin, NPA Narcissus pseudonarcissus agglutinin, $P H A-E$ phytohemagglutinin-E, PHA-L phytohemagglutinin-L, PNA peanut agglutinin, PSA Pisum sativum agglutinin, PTL-I Psophocarpus tetragonolobus lectin I, $P W M$ pokeweed mitogen, $R C A 120$ Ricinus communis agglutinin 120 , SBA soybean agglutinin, SNA Sambucus nigra agglutinin, SSA Sclerotinia sclerotiorum agglutinin, TJA-I Trichosanthes japonica agglutinin I, TJA-II Trichosanthes japonica agglutinin II, UEA-I Ulex europaeus agglutinin I, VVA Vicia villosa agglutinin, WFA Wisteria floribunda agglutinin, WGA wheat germ agglutinin

$5 \mathrm{~min}$, and the pellet was rinsed with $200 \mu \mathrm{L}$ of phosphatebuffered saline. The pellet was suspended in $20 \mu \mathrm{L}$ of phosphate-buffered saline containing $0.5 \%$ Nonidet P-40 and then sonicated gently. The suspension was stored on ice for $1 \mathrm{~h}$, and the cell debris was pulled down by centrifugation at 20,000 $\mathrm{g}$ for $5 \mathrm{~min}$. The supernatant was incubated with $10 \mu \mathrm{g}$ of Cy3-succimidyl ester (Amersham Biosciences, Tokyo, Japan) for $1 \mathrm{~h}$ at $20{ }^{\circ} \mathrm{C}$. The reaction product was applied to a Sephadex G25 spin column to remove the excess fluorescent reagent. The product was eluted by addition of $50 \mu \mathrm{L}$ of probing buffer [1.0\% Triton $\mathrm{X}-100$ and $500 \mathrm{mM}$ glycine in tris(hydroxymethyl)aminomethane-buffered saline] to the column and centrifugation at $1500 \mathrm{~g}$ for $1 \mathrm{~min}$. The collected solution was adjusted to $200 \mu \mathrm{L}$ with the probing buffer and further incubated for $2 \mathrm{~h}$ at room temperature. 


\section{Lectin microarray}

The lectin microarray which harbors 43 kinds of lectins was prepared as previously described [7, 18] (Fig. 1b). We were unable to analyze Solanum tuberosum lectin and Urtica dioica agglutinin owing to poor commercial availability during the study period, so 41 kinds of lectins were used for clinical sample assessment and were spotted on an epoxy-coated glass slide (Schott, Mainz, Germany) using a noncontact microarray printing robot (MicroSys 4000 system; Genomic Solutions, Ann Arbor, MI, USA). After the spotting, excess nonimmobilized lectins were removed by washing them with $10 \mathrm{mM}$ tris(hydroxymethyl) aminomethane-buffered saline $(\mathrm{pH} 7.4)$ containing $1 \%$ $(\mathrm{v} / \mathrm{v})$ Triton $\mathrm{X}$. The glass slide was incubated in a chamber (more than $80 \%$ humidity) at $25^{\circ} \mathrm{C}$ for $18 \mathrm{~h}$ for lectin immobilization. After incubation, a silicone rubber sheet with seven wells was carefully attached to the glass slide, and blocking was performed with $100 \mu \mathrm{L}$ of blocking solution (StabiGuard Choice; SurModics, Eden Prairie, MN, USA) at $20^{\circ} \mathrm{C}$ for $1 \mathrm{~h}$. Each lectin was spotted in triplicate as shown in Fig. 1c.

\section{Lectin array hybridization}

Cy3-labeled glycoproteins with probing buffer were added to each well of a glass slide $(60 \mu \mathrm{L}$ per well $)$ and incubated at $20{ }^{\circ} \mathrm{C}$ for $12 \mathrm{~h}$. After the unbound proteins had been washed away, lectin-bound glycoproteins were detected with an evanescent-field fluorescence scanner (GlycoStation Reader; GP BioSciences, Yokohama, Japan) in Cy3 mode. Data were analyzed with Array-Pro Analyzer version 4.5 (Media Cybernetics, Bethesda, MD, USA). The net intensity was calculated by subtracting the background from the signal intensity. A representative lectin array display is shown in Fig. 1d.

\section{Histochemical lectin staining}

Sections $(5-\mu \mathrm{m}$ thick) were cut from archival formalinfixed, paraffin-embedded tissue blocks, deparaffinized, and dehydrated through a graded series of ethanol. Endogenous peroxidase activity was quenched with $3 \%$ hydrogen peroxide-methanol. The sections were incubated with $V i$ cia villosa agglutinin (VVA; J-Oil Mills, Tokyo, Japan) at $10 \mu \mathrm{g} / \mathrm{mL}$ and with phytohemagglutinin-L (PHA-L; J-Oil Mills) at $10 \mu \mathrm{g} / \mathrm{mL}$ overnight at $4{ }^{\circ} \mathrm{C}$. Bound biotinylated lectins were then detected by the biotin-streptavidin-peroxidase complex method with a commercial kit (Vectastain ABC Elite kit; Vector Laboratories, Burlingame, CA, USA). 3,3'-Diaminobenzidine was used as the chromogen, and hematoxylin was used as a counterstain.

\section{Statistical analysis}

Statistical computations were performed using JMP version 11 (SAS Institute, Cary, NC, USA). The chi square test or the Mann-Whitney $U$ test was used to statistically analyze categorical variables, and Student's $t$ test was used to analyze continuous variables. For continuous variables, the data are expressed as the mean \pm the standard deviation. A result was considered significant if it had a $P$ value of less than $5 \%$. The time of follow-up was calculated from the operation date. Disease-specific survival was estimated according to the Kaplan-Meier method and compared using the log-rank test. A multivariate Cox proportional hazards model was built using the variables that had prognostic potential according to the univariate analysis $(P<0.1)$

\section{Results}

\section{Selection of the optimal internal control lectin}

The amount of protein from the tissues was so small (Fig. 1a) that we first explored the optimal internal control lectin candidates. We isolated protein from the seven gastric cancer cell lines (GCIY, AZ521, KE97, SH10, MKN7, MKN74, and KATO-III), and subjected the same amount (15 ng) of protein samples to lectin array analysis (Fig. S1a). As a result, Lycopersicon esculentum lectin (LEL) or Datura stramonium agglutinin (DSA) was deemed to be the most appropriate internal control lectins among the 41 lectins, because the strongest signals with minimal signal differences between cancer cells were obtained (Fig. S1). There were several lectins that had zero signal in all seven gastric cancer cell lines: Griffonia simplicifolia lectin I (GSL-I), GSL-I isoform A4 (GSL-IA4), GSL-I isoform B4 (GSL-IB4), Griffonia simplicifolia lectin II (GSL-II), peanut agglutinin (PNA), Helix pomatia agglutinin (HPA), VVA, soybean agglutinin (SBA), and Psophocarpus tetragonolobus lectin I (PTL-I) (Fig. S1a). Throughout this article we tentatively designate them as not-expressed-in-cancer (NEC) lectins, and we call other the lectins expressed-in-cancer (EC) lectins.

\section{Quantitative glycan profiling revealed reduced signals of the specific lectins in AGC with LNM}

With use of LEL as the internal control, comprehensive glycan profiles were obtained in 242 primary AGC tissues. We preliminarily examined lectin signals and clinicopathological factors by analysis of variance, and found that there was the strongest association of the specific lectin 
Fig. 2 Association of specific glycan signals with lymph node metastasis $(L N M)$ in advanced gastric cancer. a The specific not-expressed-in-cancer (NEC) lectin signals were different between N0 and N1 status. Peanut agglutinin (PNA), Griffonia simplicifolia lectin I isoform A4 (GSL-IA4), Vicia villosa agglutinin (VVA), and soybean agglutinin $(S B A)$ signal values were calculated after adjustment for Lycopersicon esculentum lectin (LEL). b The specific expressed-in-cancer (EC) lectins [Lotus tetragonolobus lectin (LTL), Erythrina crista-galli agglutinin (ECA), phytohemagglutinin- $\mathrm{L}$ (PHA-L), and Maackia amurensis lectin I (MAL-I)] were different between $\mathrm{NO}$ and N1 status. The signals were adjusted for LEL. $\mathbf{c}$ Wheat germ agglutinin (WGA) was not associated with LNM progression. The signal was adjusted for LEL. d Dot plots obtained by 2D and 3D (insert) expression of LEL, Datura stramonium agglutinin (DSA), and WGA, the internal control candidates. Confidence ellipsoids (95\%) are shown with a red line in the $2 \mathrm{D}$ plots and with a blue line in the $3 \mathrm{D}$ plot. Nonparametric densities are shown in either blue or red accordingly

\section{a NEC lectins associated with LNM}
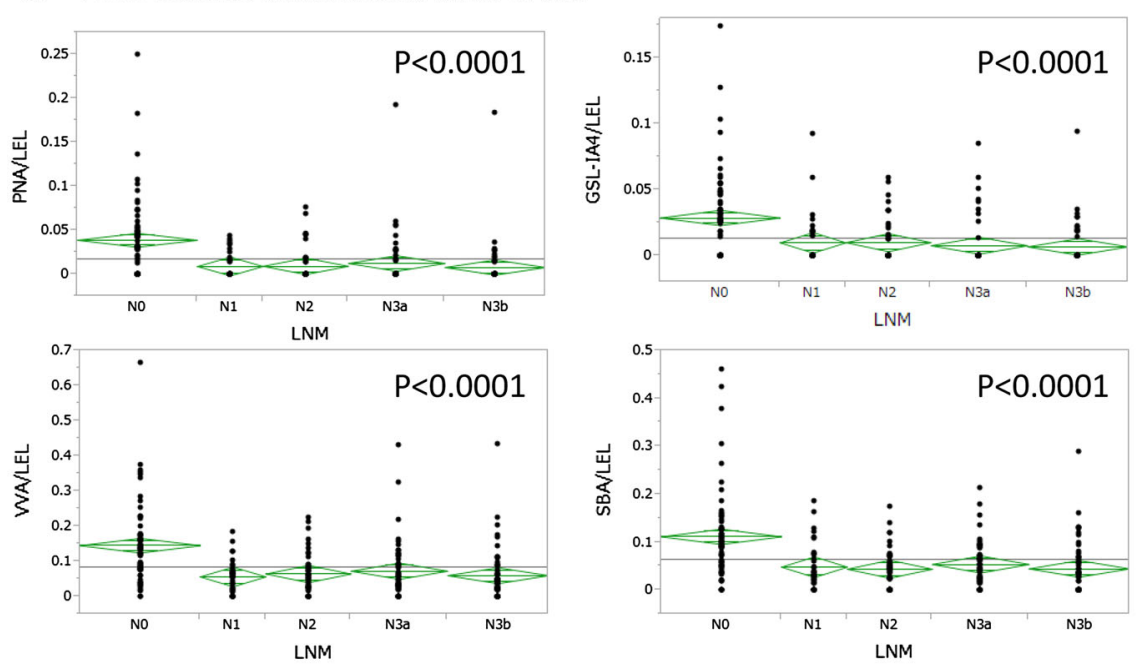

b EC lectins associated with LNM
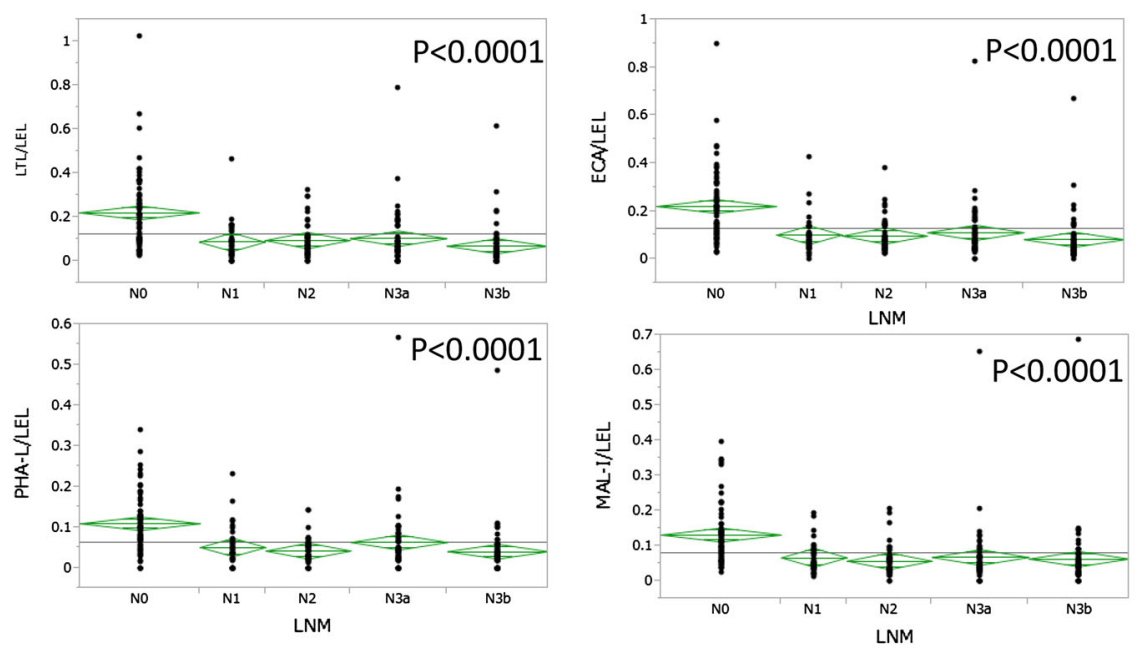

C Lectins not associated with LNM

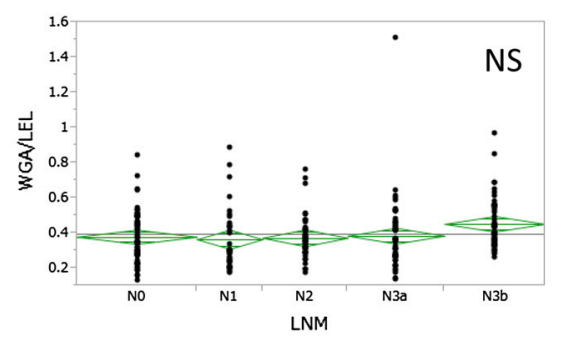

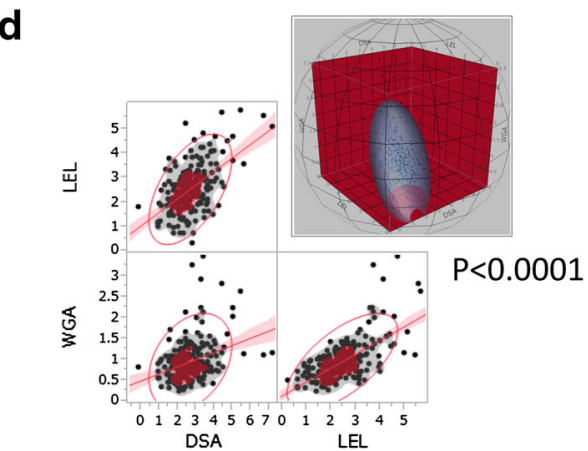

signals with $\mathrm{pN}$ status (LNM). Among the NEC lectins, a robust difference in the lectin reactive signals was found for PNA, GSL-IA4, VVA, and SBA $(P<0.0001)$ (Fig. 2a). As the NEC lectins showed zero expression in gastric cancer cell lines, expression of the NEC lectins in primary AGC tissues may have reflected the profiles of stromal cells or cancer cells affected by carcinoma-stromal cell interaction. Among the EC lectins, a robust difference in the reactive signals was also recognized for Lotus tetragonolobus lectin (LTL), Erythrina crista-galli agglutinin (ECA), PHA-L, and Maackia amurensis lectin I (MAL-I) $(P<0.0001)$ (Fig. 2b). On the other hand, no significant difference in the reactive signals was found for wheat germ agglutinin (WGA; Fig. 2c). We also 
similarly analyzed these data by using other lectins (DSA and WGA) as internal controls, because the three candidate lectins LEL, DSA, and WGA as the internal controls were relatively constantly unrelated to LNM status (Fig. 2c), and were mutually correlated (Fig. 2d).

\section{Glycan profiling of the primary AGC tissues as compared with the corresponding normal mucosa tissues}

We then examined ten gastric cancer tissues as well as the ten corresponding normal gastric mucosa tissues by lectin microarray. As a whole, reactive signals of the lectins associated with LNM were increased in the primary AGC tissues as compared with the corresponding normal tissues (Fig. S2). A significant difference in the lectin reactive signals between the primary cancer tissues and the corresponding mucosal tissues was found for Aleuria aurantia lectin (AAL; $P=$ 0.0048), Hippeastrum hybrid lectin (HHL; $P=0.0068$ ), Maackia amurensis hemagglutinin (MAH; $P=0.019$ ), and concanavalin A (Con A; $P=0.033$ ) (Fig. S3).

\section{Lectin signal profiles in AGC in terms of LNM, and determination of the optimal cutoff value to discriminate LNM}

The profiles of the LNM-associated NEC lectins (N-NEC lectins) may represent cancer-stroma interaction, because
Fig. 3 Lectin signal profiles obtained with the optimal cutoff value of the lymph mode metastasis (LNM)-associated not-expressed-in-cancer $(N$ $N E C)$ lectins. a Lectin profiles of the N-NEC lectins. The lectin signal profiles were constructed using six N-NEC lectins [Griffonia simplicifolia lectin I (2), Vicia villosa agglutinin (VVA; 3), soybean agglutinin (4), Griffonia simplicifolia lectin I isoform B4 (6), Griffonia simplicifolia lectin I isoform A4 (8), and peanut agglutinin (9) as shown in Fig. S4] and eight LNM-associated expressed-in-cancer $(N-E C)$ lectins [Maackia amurensis lectin I (10), Lotus tetragonolobus lectin (11), Erythrina crista-galli agglutinin (12), Pisum sativum agglutinin (13), Lens culinaris agglutinin (14), phytohemagglutinin-L (PHA-L; 15), Maackia amurensis hemagglutinin (16), and Hippeastrum hybrid lectin (17) as shown in Fig. S4]. White boxes represent values lower than the cutoff value. b Lectin profiles of VVA in advanced gastric cancer

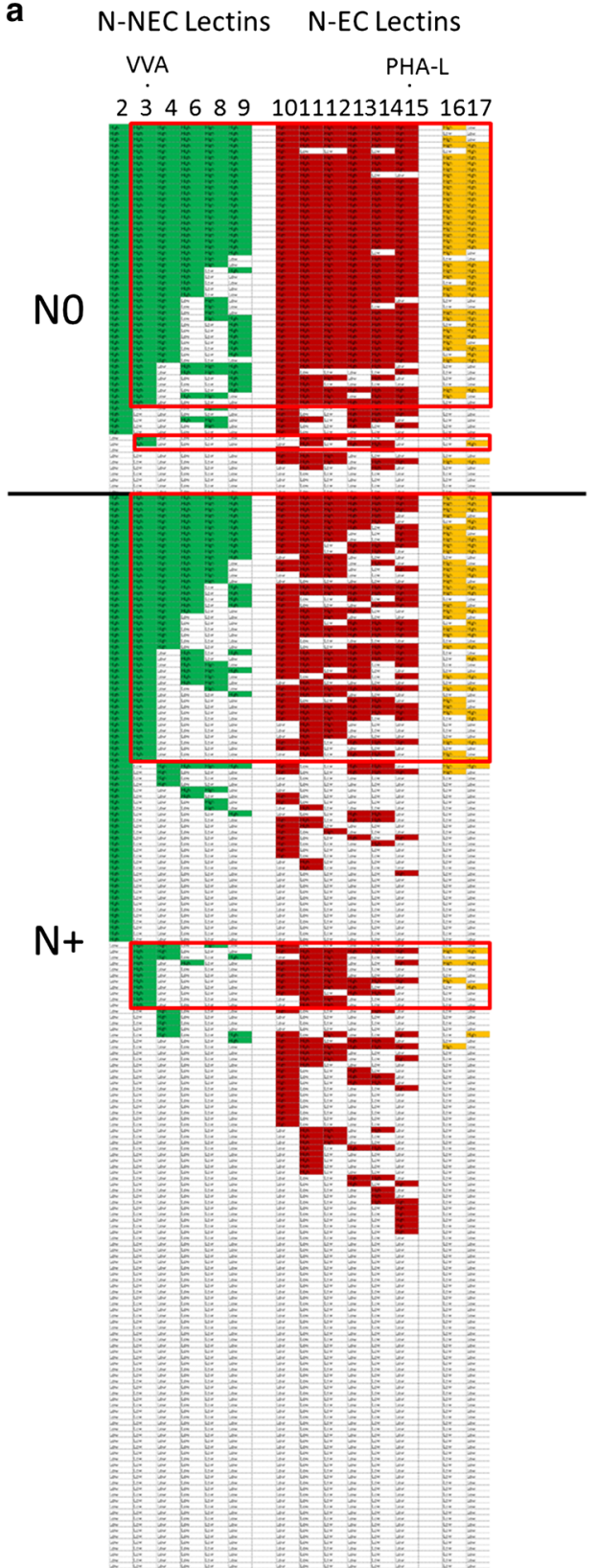


N-NEC lectins did not exhibit any signals in gastric cancer cell lines. So we constructed the lectin signal profile by using the nine N-NEC lectins (present signals of the N-NEC lectins are represented as green boxes in Fig. S4a). The profiles of LNM-associated EC lectins (N-EC lectins) are also shown in Fig. S4a (red boxes), among which the lectins were significantly overexpressed in cancer (HHL and $\mathrm{MAH}$ ) are represented as orange boxes. White boxes in Fig. S4a represent zero signal obtained by lectin array. Zero signal was frequently found for the N-NEC lectins, whereas it was seldom found for the N-EC lectins. Such zero expression of the N-NEC lectins is more frequent in AGC patients with $\mathrm{N}+$ status (pN1-3b) than in those with N0 status.

We then explored the optimal cutoff value of lectin signals to discriminate $\mathrm{N}+$ status from $\mathrm{N} 0$ status (Table $\mathrm{S} 1$ ), because the selected lectins were all involved in LNM according to Student's $t$ test as earlier described. For this purpose, we constructed the receiver operating characteristic curve to determine the optimal cutoff value (Fig. S4b). With use of these cutoff values to discriminate $\mathrm{N}+$ cases from N0 cases for individual lectins, a significant difference $(P<0.0001)$ was found between $\mathrm{N} 0$ and $\mathrm{N}+$ status in both N-EC lectins (Fig. S4c) and N-NEC lectins (Fig. S4d).

\section{Lectin signal profiles determined by the optimal cutoff value to discriminate LNM status by means of N-NEC lectins}

Lectin signal profiles of AGC in terms of LNM were reevaluated by use of the optimal cutoff value (Fig. 3a). The white boxes in Fig. 3 represent a value lower than the cutoff value. The profiles of N-NEC lectins were strongly associated with those of N-EC lectins. The most intriguing finding was that high-level signals of N-EC lectins (both red boxes and orange boxes) were almost consistent with those of VVA among the N-NEC lectins (see the red rectangles in Fig. 3). VVA was therefore rearranged in Fig. 3b. The profile of VVA was strongly associated with the profiles of almost all N-EC lectins, and was clearly correlated with $\mathrm{N}+$ status.

\section{Prognostic analysis of VVA/PHA-L in AGC}

LNM is a critical prognostic factor in AGC, so we evaluated prognosis by the log-rank test for each lectin in AGC (Fig. S5a). Among the N-EC lectins and the N-NEC lectins, PHA-L showed the most significant difference in prognosis in AGC $(P=0.001$, Fig. S5b). VVA status showed a marginal difference in prognosis in AGC $(P=0.101$, Fig. S5b). The correlation of both lectins was analyzed (Fig. S5c). VVA was strongly associated with PHA-L in both pN0 cases $(P<0.0001)$ and $\mathrm{pN}+$ cases
$(P<0.0001)$, and $\mathrm{VVA}^{+(\text {high })} \mathrm{PHA}^{-\mathrm{L}^{+(\text {high })}}$ was associated with N0 status and $\mathrm{VVA}^{-}{ }^{-} \mathrm{PHA}-\mathrm{L}^{-}$was associated with $\mathrm{N}+$ status.

We then examined the clinicopathological characteristics of PHA-L (Table S2) and VVA (Table S3) in AGC. Both lectins were closely associated with Lauren histological type, LNM, peritoneal lavage cytology test result, and growth patterns, and LNM was intensely associated with their expression. The multivariate prognostic analysis revealed that both lectins were not prognostic factors independently of LNM and the cytology test result (data not shown). These findings suggested that the contribution of PHA-L and VVA to prognosis was mediated by their LNM involvement.

\section{Reaction to VVA and PHA-L in AGC tissues}

We then investigated the reaction of VVA and PHA-L with the 20 primary AGC tissues. When we performed VVA staining, we unexpectedly saw the lectin reaction almost exclusively in cancer cells, although VVA is an NEC lectin (Fig. 4a). We also simultaneously performed immunostaining of CD68 (macrophage marker) and CD3 (T lymphocyte marker), and performed staining of PHA-L in 20 primary AGC tissues. As a result, PHA-L reaction was found in almost all types of cells, in contrast to VVA reaction. $\mathrm{CD}^{+}$cells were more frequent in VVA-positive primary AGC than in VVA-negative primary AGC; however there was no statistically significant difference (Fig. 4b).

We also performed lectin staining for 20 cases in microarray-VVA-high cases $(n=10)$ and microarray-VVAlow cases $(n=10)$ (Table 2). VVA reaction was definite in eight of the ten microarray-VVA-high patients, whereas none of the ten microarray-VVA-low patients were reactive to VVA $(P=0.00026)$. The two microarray-VVAhigh patients with no VVA reaction were lymph node positive (Table 2).

We next investigated three patients who were microarray VVA high and VVA reaction positive and who had positive LNM (Table 2). VVA reaction was negative as the metastatic lymph node station progressed in all three cases (Fig. 4c, Table 2). These findings suggested that VVA-reaction-negative cancer cells can more easily escape from tumor immunity elicited by lymphocytes than can VVAreactive cancer cells.

\section{Lectins significantly associated with Lauren histological type and p53 mutation status in AGC}

We also investigated significant association of the specific lectins with Lauren histological type and p53 mutation status in AGC (Fig. 5). The lectins significantly associated with histological type were SBA $(P=0.0037)$, Bauhinia 
VVA lectin staining

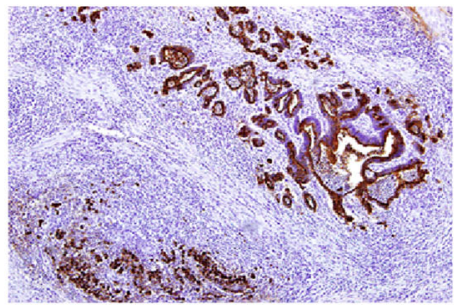

CD3 staining

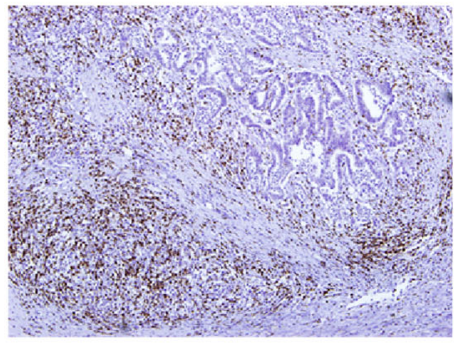

CD68 staining

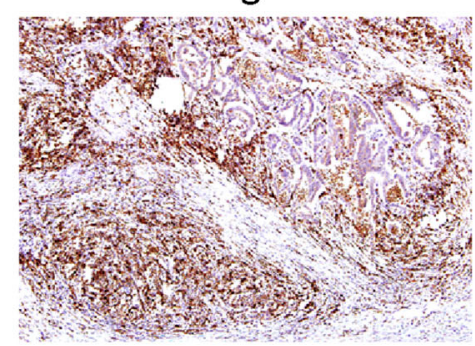

PHA-L staining

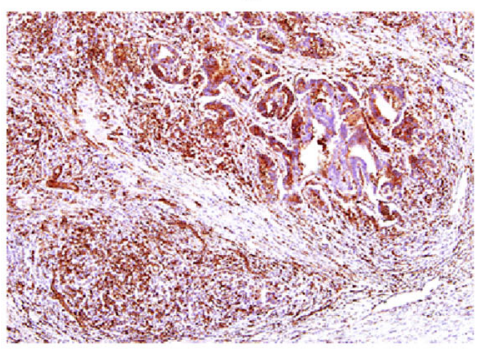

C

Case 1

LN \#1

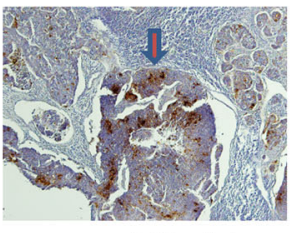

LN \#4

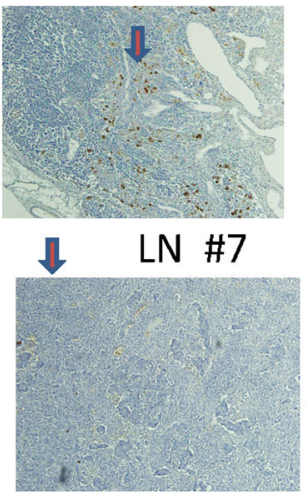

Case 2

LN \#5

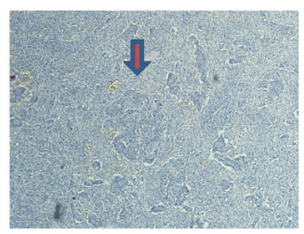

Case 3

LN \#3

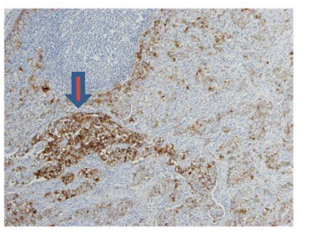

LN \#7

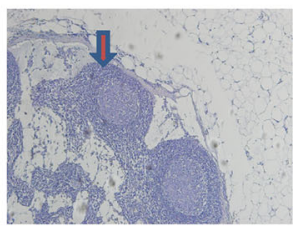

b

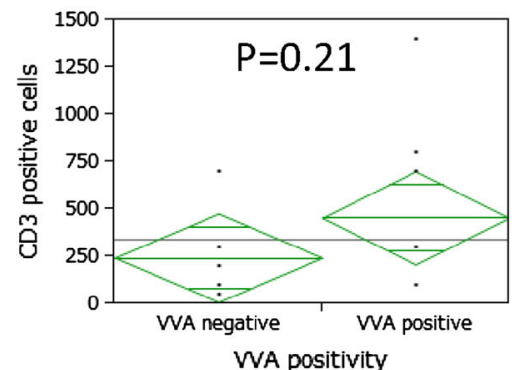

Fig. 4 Immune localization of Vicia villosa agglutinin (VVA) and its association with progression of lymph node metastasis. a VVA is clearly localized exclusively on cancer cells. Marked infiltration of macrophages (representing CD68 immunostaining) and $\mathrm{T}$ cells (representing CD3 immunostaining) was found, and phytohemagglutinin-L $(P H A-L)$ was localized both on the tumor cells and on the

purpurea lectin (BPL; $P=0.0047), \quad$ GSL-IA4 $(P=0.0116)$, VVA $(P=0.0131)$, PNA $(P=0.0137)$, PHA-L $(P=0.0174)$, GSL-IB4 $(P=0.0195)$, Wisteria floribunda agglutinin (WFA) $(P=0.0209), \quad \mathrm{HHL}$ $(P=0.0289)$, GLS-I $(P=0.0294)$, Con A $(P=0.0301)$, Ricinus communis agglutinin 120 (RCA120) $(P=0.0338)$, LTL $(P=0.0342)$, Trichosanthes japonica agglutinin II (TJA-II $)(P=0.0345)$, ECA $(P=0.0372)$, PTL-I $(P=0.0394), \quad$ Galanthus nivalis agglutinin (GNA) $(P=0.0415)$, and MAL-I $(P=0.0423)$ (Table S4).

We identified the patients who were actually investigated for p53 mutation status and described in the pathology report by routine single-strand conformation polymorphism analysis. As a result, among 253 patients, p53 mutation was investigated in 49 patients (seven mutations were identified). The lectins significantly associated with p53 mutation were GSL-II $(P<0.0001)$, PNA tumor stromal infiltrating cells. b $\mathrm{CD}^{+}$cells were more frequent in VVA-positive primary advanced gastric cancer than in VVA-negative advanced gastric cancer; however, there was no statistically significant difference. c There was no VVA in cancer cells as the metastatic lymph node station progressed in these three cases. $L N$ lymph node

$(P=0.0058)$, GSL-IA4 $(P=0.0096)$, PTL-I $(P=0.017)$, GSL-I $(P=0.0211)$, GSL-IB4 $(P=0.0336)$, HHL $(P=$ $0.0398)$, VVA $(n=0.0401)$, and TJA-II $(P=0.041)$.

\section{Discussion}

Lectin array analysis allowed very accurate quantitative assessment in minute samples in this study, whereas classical methods such immunostaining were unable to assess such delicate quantitative differences. When we investigated PHA-L reaction in tumor tissues, it was very difficult to judge whether it was high or not, because it was immunestained in almost all types of cells, so quantitative stratification was almost impossible. Our current quantitative analysis reproducibly and accurately quantified the level of individual lectin-bound glycans, and revealed strong 
Table 2 Vicia villosa agglutinin (VVA)/Lycopersicon esculentum lectin $(L E L)$ and VVA immunochistochemistry (IHC) associated with lymph node metastasis in gastric cancer

\begin{tabular}{|c|c|c|c|c|}
\hline Lectin array_VVA/LEL & VVA IHC & 14th JCGC pT & 14th JCGC pN & Involved lymph node stations \\
\hline 0.67 & + & T3 & No & \\
\hline 0.38 & + & $\mathrm{T} 4 \mathrm{a}$ & No & \\
\hline 0.36 & + & $\mathrm{T} 3$ & No & \\
\hline 0.35 & + & $\mathrm{T} 2$ & No & \\
\hline 0.35 & + & $\mathrm{T} 4 \mathrm{~b}$ & No & \\
\hline 0.43 & - & $\mathrm{T} 4 \mathrm{a}$ & N3b & 3.6 \\
\hline 0.43 & - & $\mathrm{T} 4 \mathrm{a}$ & $\mathrm{N} 2$ & $4 d, 8$ \\
\hline 0.32 & + & $\mathrm{T} 4 \mathrm{~b}$ & $\mathrm{~N} 3 \mathrm{~b}$ & $3,4 \mathrm{~d}, 7$ \\
\hline 0.22 & + & $\mathrm{T} 4 \mathrm{a}$ & $\mathrm{N} 3 \mathrm{~b}$ & $1,3,4 \mathrm{~d}, 7,9,10,11,16$ \\
\hline 0.23 & + & $\mathrm{T} 4 \mathrm{~b}$ & $\mathrm{~N} 2$ & 3,5 \\
\hline 0 & - & $\mathrm{T} 4 \mathrm{a}$ & $\mathrm{N} 3 \mathrm{~b}$ & \\
\hline 0 & - & $\mathrm{T} 4 \mathrm{a}$ & $\mathrm{N} 3 \mathrm{~b}$ & \\
\hline 0 & - & $\mathrm{T} 4 \mathrm{~b}$ & $\mathrm{~N} 2$ & \\
\hline 0 & - & $\mathrm{T} 3$ & $\mathrm{~N} 2$ & \\
\hline 0 & - & $\mathrm{T} 3$ & $\mathrm{~N} 3 \mathrm{~b}$ & \\
\hline 0 & - & $\mathrm{T} 4 \mathrm{a}$ & $\mathrm{N} 3 \mathrm{~b}$ & \\
\hline 0 & - & $\mathrm{T} 4 \mathrm{a}$ & $\mathrm{N} 3 \mathrm{a}$ & \\
\hline 0 & - & $\mathrm{T} 4 \mathrm{a}$ & N3b & \\
\hline 0 & - & $\mathrm{T} 4 \mathrm{a}$ & $\mathrm{N} 3 \mathrm{a}$ & \\
\hline 0 & - & $\mathrm{T} 4 \mathrm{a}$ & N3b & \\
\hline
\end{tabular}

JCGC Japanese Classification of Gastric Cancer correlation of the specific N-NEC lectins with N-EC lectins in AGC (Fig. 3). This study is therefore thought to involve the first successful use of quantitative glycan profiles to differentiate specific phenotypes of human cancer tissues.

The tumor tissue we used in this study was so minute that an internal control was selected on the basis of the data from gastric cancer cell lines. LEL was one of the internal control candidates, but its selection is, of course, not necessarily guaranteed for primary tumor tissues. We also used other candidates for internal controls such as DSA and WGA. The advantage of using DSA was similar to that of using LEL; DSA binding signal intensity was sufficient with minimum variation in the seven gastric cancer cell lines. With use of this internal control, the result was similar to that obtained with LEL as the internal control (Fig. S6). WGA was not significantly associated with $\mathrm{N}$ status of the primary tumor tissues, if adjustment is made for LEL (see Fig. 2), and was also applied as the internal control candidate, and similar findings were again obtained (Fig. S7). We therefore strongly suggest that the specific lectin binding signals were significantly reduced in AGC with LNM. Finally, we also compared the three candidates for the internal controls (LEL, DSA, and WGA), and found they were closely associated in terms of expression signals in primary tumor tissues (Fig. 2d). Thus, we believe that these three lectins may be appropriate as internal control lectins in investigations of primary AGC tissues.
In the beginning, we tentatively analyzed N-NEC lectins and N-EC lectins separately, because NEC lectins were presumed to reflect stromal effects of the tumor tissues, because they are never expressed in cancer cell lines. In the lectin staining in the current study, however, VVA, the most representative N-NEC lectin reflecting profiles of the N-EC lectins, clearly predominantly reacted with cancer cells (Fig. 4). VVA reaction on cancer cells may therefore be caused by carcinoma-stroma interactions. If allowance is made for expression patterns (which were overexpressed in cancer tissues and underrepresented in cancer tissues with LNM), it should reflect immunogenic status such as cancer antigenicity.

The concept of contrasuppressor $\mathrm{T}$ cell activity was introduced a quarter of a century ago, and there has been resurgence of the concept in recent research on immunoregulation [19]. VVA reaction was demonstrated to be a good marker of contrasuppressor T cells [20], and its activity was reported to be causative in collagen diseases [21] such as SLE [22]. Its activity can antagonize immune suppressor activity to augment helper $\mathrm{T}$ cell function, resulting in accelerated cancer immunity. Regulatory $\mathrm{T}$ cells (immune inhibitory $\mathrm{T}$ cells) were found to be modulated by contrasuppressor activity through specific molecules such as cytotoxic T-lymphocyte-associated protein 4 and programmed cell death 1 [23, 24], and modification of contrasuppressor activity was recently successful for human 
a histology and significantly associated lectins

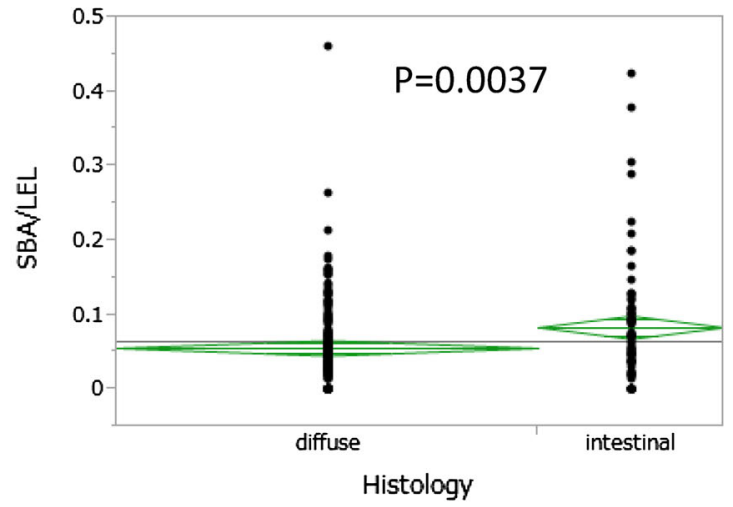

b p53 status and significantly associated lectins

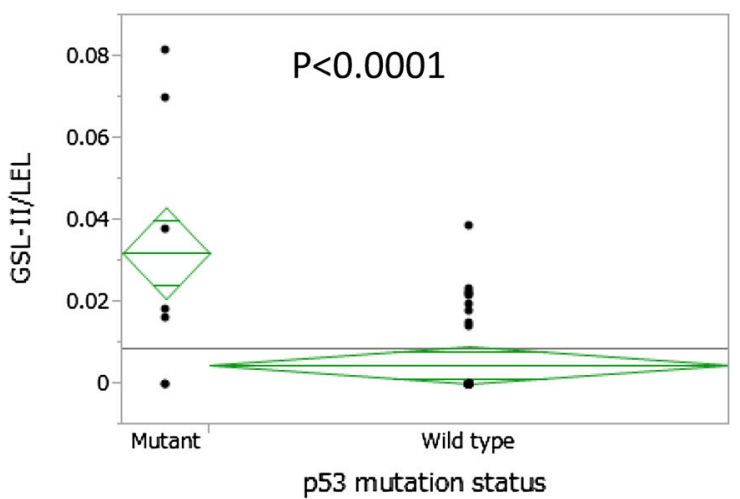

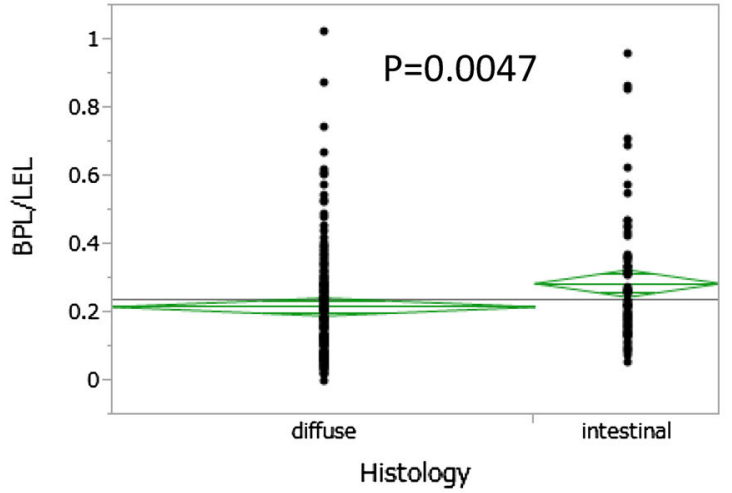

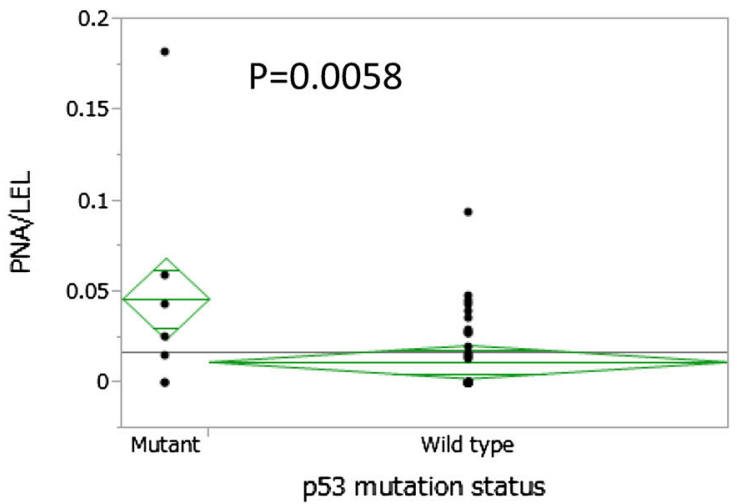

Fig. 5 Specific glycan signals associated with Lauren histological type (a) and p53 mutation status (b) in advanced gastric cancer. BPL Bauhinia purpurea lectin, GSL-I Griffonia simplicifolia lectin I, LEL Lycopersicon esculentum lectin, PNA peanut agglutinin, SBA soybean agglutinin

cancer control $[25,26]$. This is an interesting story which may explain a biological role of VVA.

However VVA-reactive $\mathrm{T}$ cells may be a minor population among the T cells in our study, and VVA reaction was found predominantly in cancer cells rather than in $\mathrm{T}$ cells. Tn antigen is composed of a carbohydrate chain, $\alpha-N$-acetyl-D-galactosamine-serine/threonine, one of the simplest glycan structures, and binds specifically to VVA. Expression of Tn antigen detected by VVA was recently reported, with a decrease in intensely labeled cell count in the most advanced stages of breast cancer [27]. This result is consistent with our quantitative analysis. Our immunohistochemistry study revealed that VVA-reactive cancer cells are almost always accompanied by remarkable infiltration of either macrophages or $\mathrm{T}$ cells, which are often immune-stained with PHA-L (Fig. 4). However, we could not conclude in this study that reduced levels of Tn antigen represented by VVA profiles can be used to determine cancer immune status.

Our findings suggest that VVA reaction accompanied by infiltration of PHA-L-reactive immune cells into stromal tissues is characteristic of middle-stage gastric cancer. Our current data are also consistent with previous reports of
PHA-L in human cancers $[28,29]$. The response of PHAL-stimulated regional lymph node cells from patients with middle-stage gastric carcinoma was demonstrated to be significantly greater than that of cells from patients with early-stage or late-stage gastric carcinoma, indicating a close association between LNM and immunological status [29]. These data suggest that PHA-L-reactive immune cells may play a crucial role in LNM development.

We also elucidated a significant association of the specific lectins with Lauren histological type and p53 mutation status in AGC (Fig. 5). The lectins the most significantly associated with histological type were SBA and BPL. Recently, lectin microarray analysis identified a decreased affinity for SBA and BPL, which was accompanied by hepatocyte growth factor induced epithelialmesenchymal transition of $\mathrm{HuH} 7$ cells, suggesting that alteration of the lectin binding profile reflecting EMT may be associated with Lauren histological type [30]. Both SMA and BPL recognize $N$-acetyl-D-galactosamine on the glycan structure, and this is supposed to be differentially regulated by galactosyltransferases according to histology findings in human gastric cancer [31]. On the other hand, p53 mutation was the most significantly associated with 
GSL-II, which is an NEC lectin. GSL-II recognizes $N$ acetyl-D glucosamine on the glycan structure, and this is a source for hyaluronan synthesis and plays a critical role in CD44 (hyaluronan receptor) biology in human cancer [32]. Mutant p53 was recently proved to have gain of function and to be directly bound to KLF17, a tumor suppressor molecule, and augmented CD44 expression by suppressing KLF17 [33]. This intriguing finding may explain our clinical result.

Critical caution is required in this study. The definition of NEC and EC lectins was based on the patterns of binding to seven gastric cancer cell lines. Because these cancer cell lines are heterogeneous, it is inadequate to define the NEC lectins as merely not binding to these gastric cancer cell lines. In fact, both PNA and VVA are NEC lectins expressed in primary AGC, and moreover it was reported that these lectins bind to gastric cancer cells [34-36]. This designation should therefore limit this analysis.

In conclusion, for the first time we have revealed comprehensive and accurate glycan profiles of human AGC tissues by lectin array, and we have discovered the specific lectins associated with LNM. The predictive value makes it worthwhile to perform further validation and pursue practical applications of this technique. Moreover, the functional significance of such glycan alterations may lead to the development of novel therapeutic strategies against AGC and may apply to other human cancers.

Acknowledgments We sincerely appreciate the technical assistance provided by Nobuko Omichi and Sachiko Unno of the National Institute of Advanced Industrial Science and Technology and by Akemi Inoue of the Kitasato University Shool of Medicine. This research was financially supported by the Medical Glycomics project of the New Energy and Industrial Technology Development Organization.

\section{References}

1. Amado M, Yan Q, Comelli EM, Collins BE, Paulson JC. Peanut agglutinin high phenotype of activated CD8 $+\mathrm{T}$ cells results from de novo synthesis of CD45 glycans. J Biol Chem. 2004;279:36689-97.

2. Comelli EM, Sutton-Smith M, Yan Q, Amado M, Panico M, Gilmartin T, Whisenant T, Lanigan CMHS, Goldberg D, Morris HR, Dell A, Paulson JC. Activation of murine CD4+ and CD8+ $\mathrm{T}$ lymphocytes leads to dramatic remodeling of $\mathrm{N}$-linked glycans. J Immunol. 2006;177:2431-40.

3. Sharon N, Lis H. History of lectins: from hemagglutinins to biological recognition molecules. Glycobiology. 2004;14:53R62R.

4. Hirabayashi J, Hayama K, Kaji H, Isobe T, Kasai K. Affinity capturing and gene assignment of soluble glycoproteins produced by the nematode Caenorhabditis elegans. J Biochem (Tokyo). 2002;132:103-14.

5. Kaji H, Saito H, Yamauchi Y, Shinkawa T, Taoka M, Hirabayashi J, Kasai K, Takahashi N, Isobe T. Lectin affinity capture, isotope-coded tagging and mass spectrometry to identify N-linked glycoproteins. Nat Biotechnol. 2003;21:667-72.

6. Zhao J, Patwa TH, Lubman DM, Simeone DM. Protein biomarkers in cancer: natural glycoprotein microarray approaches. Curr Opin Mol Ther. 2008;10:602-10.

7. Kuno A, Uchiyama N, Koseki-Kuno S, Ebe Y, Takashima S, Yamada M, Hirabayashi J. Evanescent-field fluorescence-assisted lectin microarray: a new strategy for glycan profiling. Nat Methods. 2005;2:851-6.

8. Yamashita K, Sakuramoto S, Kikuchi S, Katada N, Kobayashi N, Watanabe M. Surgical resection of stage IV gastric cancer and prognosis. Anticancer Res. 2007;27:4381-6.

9. Yamashita K, Ooki A, Sakuramoto S, Kikuchi S, Katada N, Kobayashi N, Watanabe M. Lymph node metastasis density (ND)-factor association with malignant degree and ND40 as "non-curative factor" in gastric cancer. Anticancer Res. 2008;28:435-41.

10. Yamashita K, Sakuramoto S, Kikuchi S, Katada N, Kobayashi N, Watanabe M. Validation of staging systems for gastric cancer. Gastric Cancer. 2008;11:111-8.

11. Yamashita K, Sakuramoto S, Kikuchi S, Katada N, Kobayashi N, Watanabe M. Strong association of lymph node metastasis with intraperitoneal free cancer cell (IFCC) in advanced gastric cancer. Hepatogastroenterology. 2008;55:1873-7.

12. Yamashita K, Sakuramoto S, Katada N, Futawatari N, Moriya H, Hirai K, Kikuchi S, Watanabe M. Diffuse type advanced gastric cancer showing dismal prognosis is characterized by deeper invasion and emerging peritoneal cancer cell: the latest comparative study to intestinal advanced gastric cancer. Hepatogastroenterology. 2009;56:276-81.

13. Hippo Y, Taniguchi H, Tsutsumi S, Machida N, Chong JM, Fukayama M, Kodama T, Aburatani H. Global gene expression analysis of gastric cancer by oligonucleotide microarrays. Cancer Res. 2002;62:233-40.

14. Weiss MM, Kuipers EJ, Postma C, Snijders AM, Siccama I, Pinkel D, Westerga J, Meuwissen SG, Albertson DG, Meijer GA. Genomic profiling of gastric cancer predicts lymph node status and survival. Oncogene. 2003;22:1872-9.

15. Japanese Gastric Cancer Association. Japanese classification of gastric carcinoma (English version). The 13th edition Gastric Cancer. 1998;1:10-24.

16. Matsuda A, Kuno A, Ishida H, Kawamoto T, Shoda JI, Hirabayashi J. Development of an all-in-one technology for glycan profiling targeting formalin-embedded tissue sections. Biochem Biophys Res Commun. 2008;370:259-63.

17. Matsuda A, Kuno A, Kawamoto T, Matsuzaki H, Irimura T, Ikehara Y, Zen Y, Nakanuma Y, Yamamoto M, Ohkohchi N, Shoda J-I, Hirabayashi J, Narimatsu H. Wisteria floribunda agglutinin-positive MUC1 is a sensitive biliary marker for human intrahepatic cholangiocarcinoma. Hepatology. 2010;52:174-82.

18. Uchiyama N, Kuno A, Tateno H, Kubo Y, Mizuno M, Noguchi M, Hirabayashi J. Optimization of evanescent-field fluorescence-assisted lectin microarray for high-sensitivity detection of monovalent oligosaccharides and glycoproteins. Proteomics. 2008;8:3042-50.

19. Lehner T. Special regulatory T cell review: the resurgence of the concept of contrasuppression in immunoregulation. Immunology. 2008;123:40-4.

20. Raedler A, Lenz HJ, Sandgren K, de Weerth A, Enversen S, Schreiber S, Thiele HGGH. Phenotype and functional properties of Vicia villosa agglutinin (VVA) binding $\mathrm{T}$ cells in patients with Crohn's disease: detection of contrasuppressor activity in patients lacking extra-intestinal manifestations, abscesses and fistulas. Clin Exp Immunol. 1988;74:264-9.

21. Kelly CJ, Mok H, Neilson EG. The selection of effector T cell phenotype by contrasuppression modulates susceptibility to autoimmune injury. J Immunol. 1988;141:3022-8. 
22. Fortune F, Lehner T. Phenotypic expression of Vicia villosa binding $\mathrm{T}$ cell subsets, as markers of contrasuppressor cells in systemic lupus erythematosus. Clin Exp Immunol. 1988;74:100-4.

23. Sakaguchi S. Naturally arising Foxp3-expressing CD25+ CD4+ regulatory $\mathrm{T}$ cells in immunological tolerance to self and nonself. Nat Immunol. 2005;6:345-52.

24. Nishimura H, Nose M, Hiai H, Minato N, Honjo T. Development of lupus-like autoimmune diseases by disruption of the PD-1 gene encoding an ITIM motif-carrying immunoreceptor. Immunity. 1999;11:141-51.

25. Hodi FS, O'Day SJ, McDermott DF, Weber RW, Sosman JA, Haanen JB, Gonzalez R, Robert CSD, Hassel JC, Akerley W, van den Eertwegh AJ, Lutzky J, Lorigan PVJ, Linette GP, Hogg D, Ottensmeier CH, Lebbé C, Peschel C, Quirt ICJ, Wolchok JD, Weber JS, Tian J, Yellin MJ, Nichol GM, Hoos A, Urba WJ. Improved survival with ipilimumab in patients with metastatic melanoma. N Engl J Med. 2010;363:711-23.

26. Brahmer JR, Drake CG, Wollner I, Powderly JD, Picus J, Sharfman WH, Stankevich EPA, Salay TM, McMiller TL, Gilson MM, Wang C, Selby M, Taube JM, Anders R, Chen LKA, Pardoll DM, Lowy I, Topalian SL. Phase I study of single-agent antiprogrammed death-1 (MDX-1106) in refractory solid tumors: safety, clinical activity, pharmacodynamics, and immunologic correlates. J Clin Oncol. 2010;28:3167-75.

27. Konska G, Guerry M, Caldefie-Chezet F, De Latour M, Guillot J. Study of the expression of Tn antigen in different types of human breast cancer cells using VVA-B4 lectin. Oncol Rep. 2006;15:305-10.

28. Tsakraklides E, Tsakraklides V, Ashikari H, Rosen PP, Siegal FP, Robbins GF, Good RA. In vitro studies of axillary lymph node cells in patients with breast cancer. J Natl Cancer Inst. 1975;54:549-56.

29. Kojima O, Fujita Y, Oh A, Sakita M, Nishioka B, Majima S. Immunomorphologic study of regional lymph nodes in cancer: response of regional lymph node cells from gastric and colorectal cancer to PHA stimulation. Jpn J Surg. 1980;10:212-20.

30. Li S, Mo C, Peng Q, Kang X, Sun C, Jiang K, Huang L, Lu Y, Sui $\mathrm{J}$, Qin X, Liu Y. Cell surface glycan alterations in epithelial mesenchymal transition process of Huh7 hepatocellular carcinoma cell. PLoS One. 2013;8:e71273.

31. Sutherlin ME, Nishimori I, Caffrey T, Bennett EP, Hassan H, Mandel U, Mack D, Iwamura T, Clausen H, Hollingsworth MA. Expression of three UDP-N-acetyl-alpha-D-galactosamine: polypeptide GalNAc N-acetylgalactosaminyltransferases in adenocarcinoma cell lines. Cancer Res. 1997;57:4744-8.

32. Rilla K, Oikari S, Jokela TA, Hyttinen JM, Kärnä R, Tammi RH, Tammi MI. Hyaluronan synthase 1 (HAS1) requires higher cellular UDP-GlcNAc concentration than HAS2 and HAS3. J Biol Chem. 2013;288(8):5973-83.

33. Ali A, Shah AS, Ahmad A. Gain-of-function of mutant p53: mutant p53 enhances cancer progression by inhibiting KLF17 expression in invasive breast carcinoma cells. Cancer Lett. 2014;354:87-96.

34. Fischer J, Klein PJ, Vierbuchen M, Skutta B, Uhlenbruck G, Fischer R. Characterization of glycoconjugates of human gastrointestinal mucosa by lectins. I. Histochemical distribution of lectin binding sites in normal alimentary tract as well as in benign and malignant gastric neoplasms. J Histochem Cytochem. 1984;32:681-9.

35. Shue GL, Kawa S, Kato M, Oguchi H, Kobayashi T, Koiwai T, Tokoo M, Furuta S, Kanai M, Homma T. Expression of glycoconjugates in pancreatic, gastric, and colonic tissue by Bauhinia purpurea, Vicia villosa, and peanut lectins. Scand J Gastroenterol. 1993;28:599-604.

36. Huang WL, Li YG, Lv YC, Guan XH, Ji HF, Chi BR. Use of lectin microarray to differentiate gastric cancer from gastric ulcer. World J Gastroenterol. 2014;20:5474-82. 